\title{
Ruin and Deficit Under Claim Arrivals with the Order Statistics Property
}

\author{
Dimitrina S. Dimitrova ${ }^{1} \cdot$ Zvetan G. Ignatov $^{2} \cdot$ Vladimir K. Kaishev $^{1}$
}

Received: 10 July 2017 / Revised: 21 April 2018 /

Accepted: 27 August 2018 / Published online: 19 September 2018

(C) The Author(s) 2018

\begin{abstract}
We consider an insurance risk model with extended flexibility, under which claims arrive according to a point process with an order statistics (OS) property, their amounts may have any joint distribution and the premium income is accumulated following any nondecreasing, possibly discontinuous real valued function. We generalize the definition of an OS point process, assuming it is generated by an arbitrary cdf allowing jump discontinuities, which corresponds to an arbitrary (possibly discontinuous) claim arrival cumulative intensity function. The latter feature is appealing for insurance applications since it allows to consider clusters of claims arriving instantaneously. Under these general assumptions, a closed form expression for the joint distribution of the time to ruin and the deficit at ruin is derived, which remarkably involves classical Appell polynomials. Corollaries of our main result generalize previous non-ruin formulas e.g., those obtained by Ignatov and Kaishev (Scand Actuar J 2000(1):46-62, 2000; J Appl Probab 41(2):570-578, 2004; J Appl Probab 43:535-551, 2006) and Lefèvre and Loisel (Methodol Comput Appl Probab 11(3):425-441, 2009) for the case of stationary Poisson claim arrivals and by Lefèvre and Picard (Insurance Math Econom 49:512-519, 2011; Methodol Comput Appl Probab 16:885-905, 2014), for OS claim arrivals.
\end{abstract}

Keywords Order statistics point process - Appell polynomials - Hessenberg determinants · Risk process $\cdot$ Ruin probability $\cdot$ First crossing time $\cdot$ Overshoot

Mathematics Subject Classification (2010) Primary 60K30 · Secondary 60K99

Dimitrina S. Dimitrova

d.dimitrova@city.ac.uk

Zvetan G. Ignatov

ignatov@feb.uni-sofia.bg

Vladimir K. Kaishev

v.kaishev@city.ac.uk

1 Faculty of Actuarial Science and Insurance, Cass Business School City, University of London, 106 Bunhill Row, EC1Y 8TZ London, UK

2 Faculty of Economics and Business Administration, Sofia University "St Kliment Ohridski", 125 Tsarigradsko Shosse Blv., bl.3, Sofia 1113, Bulgaria 


\section{Introduction}

The ruin of an insurance company can be viewed as the event of its aggregate claim amount exceeding for the first time the aggregate premium income, modeled by a non-decreasing deterministic function. Therefore, ruin is equivalent to first crossing of an upper deterministic boundary by a stochastic process modeling the aggregate claim amount. There have been different stochastic models of first crossing and important contributions in the applied probability literature have been made by Zolotarev (1964) and Borovkov (1964), Kou and Wang (2003), Peskir (2007), Bernyk et al. (2008), Yang and Zhang (2001), Huzak et al. (2004), Garrido and Morales (2006), Bertoin et al. (2008), and Savov (2009) and Aurzada et al. (2013) to mention only a few. The joint distribution of the first crossing time and the overshoot of a Lévy process over a fixed boundary in infinite time have been considered by Doney (1991), Klüppelberg et al. (2004), Doney and Kyprianou (2006), and Eder and Klüppelberg (2009).

In risk theory, the first crossing time and the overshoot are interpreted as the ruin time and the deficit at ruin. Ruin time and deficit in a classical infinite time risk model, have been considered jointly through a defective renewal equation in terms of what is called GerberShiu function (see Gerber and Shiu $(1997,1998)$ ). Although the literature is extensive, deriving closed form results following this approach has proved difficult (see e.g. Landriault and Willmot (2009)). Recently, Ignatov and Kaishev (2016) applied a more direct approach and derived explicitly the joint distribution of the ruin time and the deficit at ruin in a finite time interval, assuming a general dependent risk model where claim arrivals form a point process with independent increments. The latter processes represent a large and flexible class including both homogeneous and non-homogeneous Poisson and negative binomial point processes. As shown by Ignatov and Kaishev (2016), the joint ruin-time-deficit distribution is elegantly expressed in terms of a new remarkable class of functions called Appell-Hessenberg type functions.

The purpose of this paper is to extend these results to the case where claims arrive according to a point process with the so called order statistics (OS) property, or simply OS point processes, defined as follows. Consider a point process $\xi$, on $(0, \infty)$, with a cumulative intensity function $\Lambda((0, z])=\Lambda(z)=E \xi[0, z]<\infty, \forall z \in(0, \infty)$, with $\xi[0, z]$ denoting the number of claims in $[0, z]$. The process $\xi$ is said to have the OS property if, given $n$ claim arrivals in a finite interval $[0, z], z>0$, the successive arrival times, $0<T_{1}<T_{2}<\ldots<T_{n}$, coincide in distribution with the order statistics of $n$ independent and identically distributed random variables with a cumulative distribution function $F_{z}(x)$, $0 \leq x \leq z, F_{z}(z)=1$.

Following the pioneering work of Nawrotzki (1962), point processes with the OS property have been studied and characterized e.g., by Holmes (1971), Westcott (1973), Crump (1975), Kallenberg (1976), Feigin (1979), Puri (1982), Liberman (1985), and Huang and Shoung (1994) and Berg and Spizzichino (2000). More precisely, Crump (1975) has shown that OS processes are Markovian and that $F_{z}(x)=\Lambda(x) / \Lambda(z)$. It has been proven by Holmes (1971) (see also Westcott (1973)) that the only OS process with independent increments is the Poisson process. It has also been shown by Feigin (1979) that an OS process

$$
\xi \stackrel{a . s .}{=} \mathcal{P}[X \Lambda(z)],
$$

where $z>0, \mathcal{P}$ is a homogeneous Poisson process with unit rate and $X$, an independent non-negative random variable. This result states that OS point processes are characterized, up to a time-scale transformation, by mixed Poisson processes. 
In risk and ruin theory, OS processes have been applied by Willmot (1989); De Vylder and Goovaerts (1999a, b); Lefèvre and Picard (2011); Lefèvre and Picard (2014) and by Sendova and Zitikis (2012) to model claim arrivals. Such OS risk models are appealing since the total number of claims, $\xi(0, z]$ in $[0, z]$, denoted also by $N(z)$, can have any distribution, depending on the insurance application.

Let us note that, in all of the afore-quoted literature, it has been assumed that the OS process of interest has unit steps at the times $T_{1}, \ldots, T_{n}$, i.e., that the underlying cdf, $F_{z}(x)$, is continuous. In what follows, we will adopt a more general definition of an OS point process in which we allow $F_{z}(x)$ to be discontinuous. Since $F_{z}(x)$ is a distribution function, it is easy to see that the limits, $F_{z}(x+)=\lim _{s \downarrow x} F_{z}(s)$ and $F_{z}(x-)=\lim _{s \uparrow x} F_{z}(s)$ exist. If $F_{z}(x)$ is right-continuous, then $F_{z}(x)=F_{z}(x+)$ and if the difference $F_{z}(x+)-F_{z}(x-) \equiv$ $F_{z}(x)-F_{z}(x-)$ differs from zero, we will say that $F_{z}(x)$ has a jump at $x$, equal to the size of that difference. Recall also that for continuous cdf $F_{z}(x-)=F_{z}(x+)=F_{z}(x)$. We can now give the following extended definition of an OS point process.

Definition 1.1 A point process $\xi$, defined on $(0, \infty)$ with any possibly discontinuous cumulative intensity function $\Lambda((0, z])=\Lambda(z)<\infty, \forall z \in(0, \infty)$, is said to have the order statistics (OS) property if for every $0<z<\infty$ and $n \geq 0$ such that $P(\xi(0, z]=n)>0$, conditional on $\xi(0, z]=n$, the consecutive arrival times, $0<T_{1} \leq \ldots \leq T_{n} \leq z$, of $\xi$ coincide in distribution with the order statistics, $X_{1, n}, \ldots, X_{n, n}$ of $n$ independent and identically distributed random variables, $X_{1}, \ldots, X_{n}$, with a cumulative distribution function $F_{z}(x)=\Lambda(x) / \Lambda(z), 0 \leq x \leq z$, with possible jumps, such that, $F_{z}(0)=0$ and $F_{z}(z)=1$, i.e., $\left(T_{1}, \ldots, T_{n}\right) \stackrel{d}{=}\left(X_{1, n}, \ldots, X_{n, n}\right)$.

Our aim in the present paper is three-fold. First, we revisit the OS risk model considered recently by Lefèvre and Picard $(2011,2014)$ under the assumption that $F_{z}(x)$ is continuous. We relax the latter assumption and, following Definition 1.1, allow $F_{z}(x)$ to have possible jump discontinuities at fixed instants in $[0, z]$, which is equivalent to allowing $\Lambda(x)$ to be discontinuous at these instants. This leads to extending further the flexibility of the OS risk model, allowing claims to arrive at random moments but also at fixed instants with non-zero probability, possibly forming clusters. This is an appealing feature both in life and non-life insurance applications (see Section 3). Second, under this generalized OS risk model, we derive a closed-form expression for the joint distribution of the ruin time and deficit at ruin, given by Theorem 2.4, which covers and extends previous ruin probability formulas, due to Ignatov and Kaishev (2000, 2004, 2006); Ignatov et al. (2001); Lefèvre and Loisel (2009) and Lefèvre and Picard (2011, 2014). Furthermore, we demonstrate that our formulas, expressed in terms of a special case of what we call Appell-Hessenberg functions (see Ignatov and Kaishev (2016)), are more explicit. They do not involve indicator functions and expectations of random quantities, such as $N(z)$ and the aggregate claim amount $S_{N(z)}$, as is the case with the non-ruin probability formulas (4.1) and (4.2) of Lefèvre and Picard (2011) which, as the authors note, require further specification (see Lefèvre and Picard (2014)). Third, we illustrate how the expression for the joint distribution of the ruin time and deficit can be applied in some particular cases of OS claim arrivals with both continuous and discontinuous cdf $F_{z}(x)$. More precisely, we revisit the three special cases considered by Crump (1975), mixed Poisson process, linear birth process with immigration equivalent to a negative binomial $N(z)$ and a linear death process implying a binomial distribution for $N(z)$. In addition, we consider also the cases when $F_{z}(x)$ is a pure jump cdf or a cdf with 
jumps and continuous parts, with potential application in risk models involving claim counts panel data.

As noted by Lefèvre and Picard (2011), OS processes are particularly suitable for modelling dependence in the claim arrivals where the arrival of a claim increases or decreases the likelihood of more claim-arrivals leading to non-overlapping clustering, see e.g. the birth and death processes considered in Bühlmann (1970). The latter are relevant in modelling the arrival of claims from a group life policy covering a closed group of individuals. It should also be mentioned that OS processes are appealing in modelling population growth where (OS) birth and death processes are a key modelling tool (see e.g., Kendall (1949) and Haccou et al. (2005)). This demonstrates the wider applicability of the broader class of (extended, cf. Definition 1.1) OS processes compared to modelling arrivals with the classical (homogeneous) Poisson process.

The paper is organized as follows. In Section 2, we prove our main result given by Theorem 2.4. For the purpose, we formulate and prove Lemmas 2.6, 2.7 and 2.9 (and also Proposition 2.3) which are of interest in their own right, establishing explicit and recurrent representations of Appell-Hessenberg functions. Corollaries 2.10, 2.11 and 2.12 of Theorem 2.4 give ruin formulas for important special cases. In Section 3, we illustrate how the results of Section 2 can be applied for some special cases of OS claim arrival processes.

\section{A Formula for $P(T \leq z, Y>y)$}

First, let us introduce some notation and specify the ruin probability model which we will be concerned with in the sequel. The amounts of consecutive claims to an insurance company are modelled by the random variables $W_{1}, W_{2}, \ldots$, and $Y_{1}, Y_{2}, \ldots$ denote their partial sums, i.e. $Y_{1}=W_{1}, Y_{2}=W_{1}+W_{2}, \ldots$ If claim severities $W_{1}, W_{2}, \ldots, W_{k}$ are considered continuous random variables, then $\psi\left(w_{1}, \ldots, w_{k}\right)$ will denote their joint density and $f\left(y_{1}, \ldots, y_{k}\right)$ will denote the joint density of $Y_{1}, Y_{2}, \ldots, Y_{k}$. Clearly, $\psi\left(w_{1}, \ldots, w_{k}\right)=$ $f\left(w_{1}, w_{1}+w_{2}, \ldots, w_{1}+\ldots+w_{k}\right)$ and $f\left(y_{1}, \ldots, y_{k}\right)=\psi\left(y_{1}, y_{2}-y_{1}, \ldots, y_{k}-y_{k-1}\right)$. In the case of discrete claim severities $W_{1}, W_{2}, \ldots, W_{k}$, their joint probability mass function is denoted by $P_{w_{1}, \ldots, w_{k}}=P\left(W_{1}=w_{1}, \ldots, W_{k}=w_{k}\right)$.

We will further assume that claims arrive according to an OS point process $\xi$, defined as in Definition 1.1 which extends the OS property considered previously in the literature.

The cumulative premium income of the insurance company up to time $t$ is modelled by the function $h(t)$ which is assumed a non-negative and non-decreasing real-valued function, defined on $[0,+\infty)$, such that $\lim _{t \rightarrow \infty} h(t)=+\infty$. Let us also note that the function $h(t)$ does not need to be necessarily continuous and can therefore model arrivals of lump sum premium amounts. If $h(t)$ is discontinuous, we define $h^{-1}(y)=\inf \{v: h(v) \geq y\}$.

We consider a finite time interval $[0, z]$, where $z$ is a fixed positive real number. We will further consider the restriction of $h(t)$ on $[0, z]$, denoted by $h_{z}(t)$, and define the corresponding restriction of the inverse function as $h_{z}^{-1}(y)=\min \left(z, h^{-1}(y)\right)$. We express the insurance company's surplus process as $R_{t}=h_{z}(t)-S_{t}$, where $S_{t}=Y_{\xi(0, t]}$ is the aggregate claim amount process, and the instant of ruin, $T$, is defined as $T:=$ $\inf \left\{t: 0<t \leq z, R_{t}<0\right\}$ or $T=\infty$ if $R_{t} \geq 0$ for all $0<t \leq z$. Given ruin occurs within $[0, z]$, i.e., $T \leq z$, the deficit at ruin, $Y$, is defined as $Y=-R_{T}$. Denote by $P(T>z)$ the probability of non-ruin in $[0, z]$, i.e., $P(T>z)=P\left(R_{t} \geq 0, \forall t \in(0, z]\right)$ and by $P(T \leq z, Y>y)$ the probability that ruin occurs before time $z$, with a deficit, $Y$, exceeding $y \geq 0$. In what follows, we will give explicit expressions for these and other related probabilities under the assumption that the process of claim arrivals, $\xi$, belongs to the class of 
point processes with the OS property, described in Definition 1.1. To reflect on the OS property of $\xi$, we will refer to the related risk model as an OS risk model. In order to formulate our main result, we will need to introduce a particular type of classical Appell polynomials which belong to the wider class of Appell-Hessenberg functions considered in Ignatov and Kaishev (2016).

Definition 2.1 For a fixed non-negative integer $j$, let $0 \equiv z_{0}<z_{1}<z_{2}<\ldots<z_{j}<$ $z_{j+1} \equiv z$ be an arbitrary increasing sequence of positive real numbers and $p_{k}=F_{z}\left(z_{k}-\right)-$ $F_{z}\left(z_{k-1}-\right), k=1,2, \ldots, j, p_{j+1}=F_{z}\left(z_{j+1}\right)-F_{z}\left(z_{j}-\right) \equiv F_{z}(z)-F_{z}\left(z_{j}-\right)$ with $p_{1}+$ $\ldots+p_{j+1}=1$. Define the functions $A_{j}\left(F_{z}(z) ; F_{z}\left(z_{1}-\right), \ldots, F_{z}\left(z_{j}-\right)\right), z \in\left(z_{j}, \infty\right)$, $j=0,1,2, \ldots$ as

$$
A_{j}\left(F_{z}(z) ; F_{z}\left(z_{1}-\right), \ldots, F_{z}\left(z_{j}-\right)\right)=(-1)^{j} \operatorname{det}\left(\left(\delta_{m, l}^{(j)}\right)_{1 \leq m, l \leq j+1}\right),
$$

where $\delta_{1,1}^{(0)} \equiv 1$ for $j=0$,

$$
\delta_{m, l}^{(j)}=\left(\begin{array}{c}
j-l+1 \\
m-l+1
\end{array}\right)\left(p_{1}+\ldots+p_{m}\right)^{m-l+1},
$$

for $1 \leq m \leq j, 1 \leq l \leq j+1$ with

$$
\begin{aligned}
& \left(\begin{array}{c}
j-l+1 \\
m-l+1
\end{array}\right) \equiv 0, \quad \text { if } \quad m-l+1<0, \\
& \left(\begin{array}{c}
j-l+1 \\
m-l+1
\end{array}\right) \equiv 1 \quad \text { if } \quad m-l+1=0,
\end{aligned}
$$

and where $\delta_{j+1, l}^{(j)} \equiv 1$ for $1 \leq l \leq j+1$.

Remark 2.2 From Eq. 3, it follows that $\left(\delta_{m, l}^{(j)}\right)_{1 \leq m, l \leq j+1}$ is a lower Hessenberg matrix. A matrix whose elements above or below the first subdiagonal are equal to zero (i.e., all elements $a_{i j}=0$ if $j-i>1$ or if $i-j>1$ ) are called Hessenberg matrixes. For properties of Hessenberg matrixes and their determinants we refer to e.g. Vein and Dale (1999). Note also that $A_{j}\left(F_{z}(z) ; F_{z}\left(z_{1}-\right), \ldots, F_{z}\left(z_{j}-\right)\right)$ is a classical Appell polynomial of degree $j$, defined by the sequence $F_{z}\left(z_{1}-\right), \ldots, F_{z}\left(z_{j}-\right)$ and evaluated at $F_{z}(z)=1$ i.e.,

$$
A_{j}\left(F_{z}(z) ; F_{z}\left(z_{1}-\right), \ldots, F_{z}\left(z_{j}-\right)\right)=A_{j}\left(1 ; F_{z}\left(z_{1}-\right), \ldots, F_{z}\left(z_{j}-\right)\right),
$$

where

$$
\begin{aligned}
A_{0}(F(z)) & =1, \\
A_{j}^{\prime}(F(z)) & =c A_{j-1}(F(z)), \text { and } \\
A_{j}\left(F\left(z_{j}-\right)\right) & =0,
\end{aligned}
$$

$j=1,2, \ldots$, with $c$, a constant and $0 \leq z_{1} \leq \ldots \leq z_{j}, z_{j} \in \mathbb{R}$.

Classical Appell polynomials, defined above, were first shown to appear in ruin theory in the closed form non-ruin probability formulas due to Ignatov and Kaishev (2000, 2004) in relation to the Poisson claim arrivals in a general risk model with dependence. It was shown by Ignatov and Kaishev (2000) (see Lemma 1 therein) that Appell polynomials can be represented as certain Hessenberg determinants. For further properties of classical 
Appell polynomials and their relation to ruin probability see Dimitrova et al. (2016). A different class of so called generalized Appell polynomials, which do not yield classical Appell polynomials, was considered by Picard and Lefèvre (1997).

Since the functions $A_{j}\left(F_{z}(z) ; F_{z}\left(z_{1}-\right), \ldots, F_{z}\left(z_{j}-\right)\right), j=1,2, \ldots$ are values of Appell polynomials expressed as Hessenberg determinants, we will more generally refer to them as Appell-Hessenberg type functions. For other types of such functions see Ignatov and Kaishev (2016).

In what follows, it will some times be convenient to interchangeably use the notation $0 \equiv z_{0}<z_{1}<z_{2}<\ldots<z_{j}<z_{j+1}$, with $z_{j+1} \equiv z$, for the sequence $0 \equiv z_{0}<z_{1}<$ $z_{2}<\ldots<z_{j}<z$. The following recurrence formula facilitates the numerical evaluation of the Appell-Hessenberg functions, $A_{j}\left(F_{z}(z) ; F_{z}\left(z_{1}-\right), \ldots, F_{z}\left(z_{j}-\right)\right)$.

Proposition 2.3 For a fixed non-negative integer $j$, let $0 \equiv z_{0}<z_{1}<z_{2}<\ldots<z_{j}<z$ be an arbitrary increasing sequence of positive real numbers. For the Appell-Hessenberg functions, $A_{j}\left(F_{z}(z) ; F_{z}\left(z_{1}-\right), \ldots, F_{z}\left(z_{j}-\right)\right)$, defined in Eq. 2, we have

$$
A_{j}\left(F_{z}(z) ; F_{z}\left(z_{1}-\right), \ldots, F_{z}\left(z_{j}-\right)\right)=\sum_{i=0}^{j} \delta_{j+1, i+1}^{(j)} A_{i}\left(0 ; F_{z}\left(z_{1}-\right), \ldots, F_{z}\left(z_{i}-\right)\right), j \geq 0,
$$

where $A_{0}\left(F_{z}(z)\right) \equiv 1, z \geq 0$ and

$$
A_{i}\left(0 ; F_{z}\left(z_{1}-\right), \ldots, F_{z}\left(z_{i}-\right)\right)=-\sum_{k=0}^{i-1} \delta_{i, k+1}^{(j)} A_{k}\left(0 ; F_{z}\left(z_{1}-\right), \ldots, F_{z}\left(z_{k}-\right)\right), i \geq 1,
$$

with $A_{0}(0) \equiv 1$.

Proof of Proposition 2.3 The proof is similar to the proof given in Ignatov and Kaishev (2000) for the case of classical Appell polynomials (see Lemma 1 therein) and is therefore omitted.

Next, we state our main result which shows that the joint distribution of the time to ruin and the deficit at ruin in the risk model with claim arrivals following an arbitrary OS point process, $\xi$, from Definition 1.1, can be expressed in terms of the Appell-Hessenberg functions, $A_{j}\left(F_{z}(z) ; F_{z}\left(z_{1}\right), \ldots, F_{z}\left(z_{j}\right)\right), j=0,1,2, \ldots$

Theorem 2.4 The probability $P(T \leq z, Y>y), 0<z<\infty, y \geq 0$, is given by

$$
\begin{aligned}
& P(T \leq z, Y>y)=P(\xi(0, z]=1) \int_{y}^{\infty}\left(1-P\left(\xi\left(0, h_{z}^{-1}\left(y_{1}-y\right)\right]=0\right)\right) f\left(y_{1}\right) d y_{1} \\
& +\sum_{j=2}^{\infty} P(\xi(0, z]=j) \sum_{k=1}^{j} \int \ldots \int\left\{A_{k-1}\left(1 ; F_{z}\left(h_{z}^{-1}\left(y_{1}\right)-\right), \ldots, F_{z}\left(h_{z}^{-1}\left(y_{k-1}\right)-\right)\right)\right. \\
& \left.-A_{k}\left(1 ; F_{z}\left(h_{z}^{-1}\left(y_{1}\right)-\right), \ldots, F_{z}\left(h_{z}^{-1}\left(y_{k-1}\right)-\right), F_{z}\left(h_{z}^{-1}\left(y_{k}-y\right)-\right)\right)\right\} \\
& f\left(y_{1}, \ldots, y_{k}\right) d y_{k} \ldots d y_{1},
\end{aligned}
$$

where $C_{k}=\left\{\left(y_{1}, \ldots, y_{k}\right): 0<y_{1}<\ldots<y_{k-1} \leq y_{k}-y, y_{k-1} \leq h_{z}(z)\right\}$, and $A_{j}\left(1 ; F_{z}\left(z_{1}-\right), \ldots, F_{z}\left(z_{j}-\right)\right)$ are the classical Appell polynomials evaluated at $F_{z}(z)=1$ and defined as in Eq. 2 with $z_{1}=h_{z}^{-1}\left(y_{1}\right), \ldots, z_{j}=h_{z}^{-1}\left(y_{j}\right), j=0,1,2 \ldots$. 
Remark 2.5 It should be noted that for the efficient numerical evaluation of $P(T \leq z, Y>$ $y$ ), following Eq. 6, it is essential to be able to: 1) appropriately truncate the infinite summation; 2) compute the underlying multiple integrals; 3 ) efficiently compute the integrand functions $A_{j}\left(F_{z}(z) ; F_{z}\left(z_{1}-\right), \ldots, F_{z}\left(z_{j}-\right)\right)$. The latter can be done using recurrence formula (5). Methods for solving 1) and 2) developed in Dimitrova et al. (2016) for the special case of stationary Poisson claim arrivals could be generalized to the case of OS claim arrivals. Details of how this could be done are outside the scope of the present paper and will be considered separately.

In order to prove Theorem 2.4 and some related corollaries, we will need the following lemmas.

Lemma 2.6 For the real sequence $0 \equiv z_{0}<z_{1}<z_{2}<\ldots<z_{j}<z_{j+1} \equiv z$, $A_{j}\left(F_{z}(z) ; F_{z}\left(z_{1}-\right), \ldots, F_{z}\left(z_{j}-\right)\right)$, defined as in Eq. 2, and $p_{k}$ introduced in Definition 2.1, we have

$$
A_{j}\left(F_{z}(z) ; F_{z}\left(z_{1}-\right), \ldots, F_{z}\left(z_{j}-\right)\right)=\sum_{\left(g_{0}, \ldots, g_{j}\right) \in E(0, j)} \frac{j !}{g_{0} ! \ldots g_{j} !} p_{1}^{g_{0}} \ldots p_{j+1}^{g_{j}}
$$

where $E(0, j)$ is the set of $(j+1)$-tuples of non-negative integers such that

$$
\begin{aligned}
E(0, j)= & \left\{\left(g_{0}, \ldots, g_{j}\right): g_{0} \equiv 0,0 \leq g_{1} \leq 1, \ldots, 0 \leq g_{1}+\ldots+g_{j-1} \leq j-1,\right. \\
& \left.g_{1}+\ldots+g_{j}=j\right\},
\end{aligned}
$$

$j \geq 0$, and where for notational convenience we assume that $z_{j+1} \equiv z$.

Proof of Lemma 2.6 We will proceed by induction. First, we verify that Lemma 2.6 holds in the cases $j=0$ and $j=1$. When $j=0$ and $0 \equiv z_{0}<z$, from Eq. 2, for the left-hand side of Eq. 7, we have $A_{0}\left(F_{z}(z)\right) \equiv 1$ and for the right-hand side, we have

$$
\sum_{g_{0} \in E(0,0)} \frac{0 !}{g_{0} !} p_{1}^{g_{0}}=1
$$

and therefore, Lemma 2.6 holds. When $j=1$ and $0 \equiv z_{0}<z_{1}<z$, from Eq. 2, for the left-hand side of Eq. 7, we have

$$
A_{1}\left(F_{z}(z) ; F_{z}\left(z_{1}\right)-\right)=(-1) \operatorname{det}\left(\begin{array}{cc}
p_{1} & 1 \\
p_{1}+p_{2} & 1
\end{array}\right)=-\operatorname{det}\left(\begin{array}{cc}
p_{1} & 1 \\
1 & 1
\end{array}\right)=1-p_{1}
$$

and for the right-hand side we have that

$$
\sum_{\left(g_{0}, g_{1}\right) \in E(0,1)} \frac{1 !}{g_{0} ! g_{1} !} p_{1}^{g_{0}} p_{2}^{g_{1}}=p_{2}=1-p_{1}
$$

and therefore, equality (7) is again valid. We will continue the proof by induction. We showed that Lemma 2.6 holds for $j=0$ and $j=1$. Assume it is true for all non-negative integers up to $j-1$. Lemma 2.6 will be proved if we show that Eq. 7 is true also for the 
index $j$. Let us expand the determinant on the right-hand side of equality (2) with respect to its first column. We have

$$
\begin{aligned}
& A_{j}\left(F_{z}(z) ; F_{z}\left(z_{1}-\right), \ldots, F_{z}\left(z_{j}-\right)\right)=(-1)^{j} \operatorname{det}\left(\left(\delta_{m, l}^{(j)}\right)_{1 \leq m, l \leq j+1}\right)=(-1)^{j}\left(\left(\begin{array}{c}
j \\
1
\end{array}\right) p_{1}^{1} A_{1,1}\right. \\
& \left.+\left(\begin{array}{c}
j \\
2
\end{array}\right)\left(p_{1}+p_{2}\right)^{2} A_{2,1}+\ldots+\left(\begin{array}{c}
j \\
j
\end{array}\right)\left(p_{1}+\ldots+p_{j}\right)^{j} A_{j, 1}+\left(p_{1}+\ldots+p_{j+1}\right)^{j} A_{j+1,1}\right),
\end{aligned}
$$

where $A_{k, 1}=(-1)^{k+1} \operatorname{det} \Delta_{k, 1}$ is the cofactor of the element $\delta_{k, 1}^{(j)}, 1 \leq k \leq j+1$, on the $k$-th row and the 1-st column of $\left(\delta_{m, l}^{(j)}\right)_{1 \leq m, l \leq j+1}$ and $\Delta_{k, 1}$ is a sub-matrix, obtained by deleting the $k$-th row and 1 -st column of $\left(\delta_{m, l}^{(j)}\right)_{1 \leq m, l \leq j+1}$. For $1<k \leq j$, we can express the matrix $\Delta_{k, 1}$ in a block-matrix form as

$$
\Delta_{k, 1}=\left(\begin{array}{ll}
\delta_{1,1} & \delta_{1,2} \\
\delta_{2,1} & \delta_{2,2}
\end{array}\right),
$$

where $\delta_{1,1}$ is a $(k-1) \times(k-1)$ unit lower triangular matrix, i.e. with ones on the main diagonal and zeros in the upper triangle, $\delta_{1,2}$ is a $(k-1) \times(j-k+1)$ matrix of zeros (hence, the matrix $\delta_{2,1}$ does not play a role in computing $\operatorname{det}\left(\Delta_{k, 1}\right)$, see Eq. 13), and $\delta_{2,2}$ is a $(j-k+1) \times(j-k+1)$ matrix for fixed $k, 1<k \leq j$, obtained from $\left(\delta_{m, l}^{(j)}\right)_{1 \leq m, l \leq j+1}$, applying in it the following formal substitutions

$$
j \rightarrow j-k ; p_{1} \rightarrow p_{1}+\ldots+p_{k+1} ; p_{2} \rightarrow p_{k+2} ; \ldots ; p_{j-k} \rightarrow p_{j} ; p_{j-k+1} \rightarrow p_{j+1} .
$$

Similarly, for $k=1$, the matrix $\Delta_{1,1}$ is defined by $\left(\delta_{m, l}^{(j)}\right)_{1 \leq m, l \leq j+1}$, applying in it the following substitutions

$$
j \rightarrow j-1 ; \quad p_{1} \rightarrow p_{1}+p_{2} ; \quad p_{2} \rightarrow p_{3} ; \ldots ; p_{j-1} \rightarrow p_{j} ; \quad p_{j} \rightarrow p_{j+1} .
$$

Since $\delta_{1,1}$ is a unit lower triangular matrix,

$$
\operatorname{det}\left(\delta_{1,1}\right)=1 \text {, }
$$

whereas by the induction assumption, for the determinant of $\delta_{2,2}$, we have

$$
(-1)^{j-k} \operatorname{det}\left(\delta_{2,2}\right)=\sum_{\left(g_{0}, \ldots, g_{j-k}\right) \in E(0, j-k)} \frac{(j-k) !}{g_{0} ! \ldots g_{j-k} !}\left(p_{1}+\ldots+p_{k+1}\right)^{g_{0}} p_{k+2}^{g_{1}} \ldots p_{j+1}^{g_{j-k}} \text {. }
$$

Hence, from Eqs. 10, 11 and 12 for $1 \leq k \leq j$, we have

$$
\begin{aligned}
& \operatorname{det}\left(\Delta_{k, 1}\right)=\operatorname{det}\left(\delta_{1,1}\right) \operatorname{det}\left(\delta_{2,2}\right) \\
& =(-1)^{j-k} \sum_{\left(g_{0}, \ldots, g_{j-k}\right) \in E(0, j-k)} \frac{(j-k) !}{g_{0} ! \ldots g_{j-k} !}\left(p_{1}+\ldots+p_{k+1}\right)^{g_{0}} p_{k+2}^{g_{1}} \ldots p_{j+1}^{g_{j-k}} \cdot(13)
\end{aligned}
$$

If $k=j$ then from Eq. 13

$$
\operatorname{det}\left(\Delta_{j, 1}\right)=1
$$

whereas for $k=j+1$ we have

$$
\operatorname{det}\left(\Delta_{j+1,1}\right)=1
$$


From Eqs. 13, 14 and 15, for the cofactors $A_{k, 1}, 1 \leq k \leq j$, and $A_{j+1,1}$ we have

$$
\begin{aligned}
A_{k, 1} & =(-1)^{k+1} \operatorname{det}\left(\Delta_{k, 1}\right) \\
& =(-1)^{j+1} \sum_{\left(g_{0}, \ldots, g_{j-k}\right) \in E(0, j-k)} \frac{(j-k) !}{g_{0} ! \ldots g_{j-k} !}\left(p_{1}+\ldots+p_{k+1}\right)^{g_{0}} p_{k+2}^{g_{1}} \ldots p_{j+1}^{g_{j-k}},
\end{aligned}
$$

and

$$
A_{j+1,1}=(-1)^{j+2} \text {. }
$$

Substituting Eqs. 16 and 17 back in Eq. 9, we obtain

$$
\begin{aligned}
& A_{j}\left(F_{z}(z) ; F_{z}\left(z_{1}-\right), \ldots, F_{z}\left(z_{j}-\right)\right)=(-1)^{j} \operatorname{det}\left(\left(\delta_{m, l}^{(j)}\right)_{1 \leq m, l \leq j+1}\right) \\
& =\left(p_{1}+\ldots+p_{j+1}\right)^{j}-\left(\left(\begin{array}{l}
j \\
1
\end{array}\right) p_{1}^{1} \sum_{\left(g_{0}, \ldots, g_{j-1}\right) \in E(0, j-1)} \frac{(j-1) !}{g_{0} ! \ldots g_{j-1} !}\left(p_{1}+p_{2}\right)^{g_{0}} p_{3}^{g_{1}} \ldots p_{j+1}^{g_{j-1}}\right. \\
& +\left(\begin{array}{c}
j \\
2
\end{array}\right)\left(p_{1}+p_{2}\right)^{2} \sum_{\left(g_{0}, \ldots, g_{j-2}\right) \in E(0, j-2)} \frac{(j-2) !}{g_{0} ! \ldots g_{j-2} !}\left(p_{1}+p_{2}+p_{3}\right)^{g_{0}} p_{4}^{g_{1}} \ldots p_{j+1}^{g_{j-2}} \\
& +\ldots+\left(\begin{array}{c}
j \\
k
\end{array}\right)\left(p_{1}+\ldots+p_{k}\right)^{k} \sum_{\left(g_{0}, \ldots, g_{j-k}\right) \in E(0, j-k)} \frac{(j-k) !}{g_{0} ! \ldots g_{j-k} !} \\
& \quad \times\left(p_{1}+\ldots+p_{k+1}\right)^{g_{0}} p_{k+2}^{g_{1}} \ldots p_{j+1}^{g_{j-k}} \\
& \left.\quad+\ldots+\left(\begin{array}{l}
j \\
j
\end{array}\right)\left(p_{1}+\ldots+p_{j}\right)^{j} \sum_{g_{0} \in E(0,0)} \frac{0 !}{g_{0} !}\left(p_{1}+\ldots+p_{j+1}\right)^{g_{0}}\right) \\
& = \\
& \quad \sum_{\left(g_{0}, \ldots, g_{j}\right) \in E(0, j)} \frac{j !}{g_{0} ! \ldots g_{j} !} p_{1}^{g_{0}} \ldots p_{j+1}^{g_{j}},
\end{aligned}
$$

where the last equality, in Eq. 18 follows after some tedious but straightforward algebra, which is therefore omitted. This completes the proof of Lemma 2.6.

Lemma 2.7 Let $0 \equiv z_{0}<z_{1}<z_{2}<\ldots<z_{j}<z$ be a sequence of positive real numbers and for a fixed $z$, let $0<T_{1} \leq T_{2} \leq \ldots \leq T_{j} \leq z$ be the consecutive points of an OS point process $\xi$, defined as in Definition 1.1. We have

$$
P\left(T_{1}>z_{1}, \ldots, T_{j}>z_{j}\right)=\sum_{\left(g_{0}, \ldots, g_{j}\right) \in E(0, j)} \frac{j !}{g_{0} ! \ldots g_{j} !} p_{1}^{g_{0}} \ldots p_{j+1}^{g_{j}},
$$

where $E(0, j)$ is the set of $(j+1)$-tuples of non-negative integers, defined in Eq. 8, and $p_{k}$, $k=1,2, \ldots, j+1$ are defined in Definition 2.1.

Remark 2.8 When $j=0$, the left-hand side of Eq. 19 should be interpreted as the conditional probability of non-ruin, given that there are zero claims in $(0, z]$, i.e., that $\xi(0, z]=0$. Clearly, this conditional probability is equal to one, and in this case equality (19) is still valid since substituting $j=0$ on the right-hand side also gives one. 
Proof of Lemma 2.7 The proof will be based on interpreting $P\left(T_{1}>z_{1}, \ldots, T_{j}>z_{j}\right)$ as the probability of non-crossing within $[0, z]$ of an upper deterministic boundary,

$$
h_{z}(x)= \begin{cases}0 & \text { for } x \in\left[0, z_{1}\right), \\ 1 & \text { for } x \in\left[z_{1}, z_{2}\right), \\ \ldots & \text { for } x \in\left[z_{j-1}, z_{j}\right), \\ (j-1) & \text { for } x \in\left[z_{j-1}, z_{j}\right],\end{cases}
$$

by the trajectory of an OS counting process, $\xi$, defined as in Definition 1.1. It is easy to see that every trajectory of $\xi$, i.e., the number of points of the random point set $X_{1}, \ldots, X_{j}$, which occur in the interval $[0, x]$, for $0<x \leq z$, coincide in distribution with $j F_{j}(x)$, i.e., $\xi[0, x] \stackrel{d}{=} j F_{j}(x)$, where $F_{j}(x)$ is the empirical distribution function based on the sample $X_{1}, \ldots, X_{j}$. Furthermore, it can directly be seen that the event

$$
\left\{\omega: T_{1}(\omega)>z_{1}, \ldots, T_{j}(\omega)>z_{j}\right\}=\left\{\omega: \xi[0, x] \leq h_{z}(x) \text {, for every } x \in(0, z]\right\},
$$

i.e., the probability of non-crossing $h_{z}(x)$, is equal to $P\left(T_{1}>z_{1}, \ldots, T_{j}>z_{j}\right)$.

On the other hand, let us interpret $X_{1}, \ldots, X_{j}$ as the consecutive random placement of $j$ independent points on the interval $[0, z]$, which is partitioned into $j+1$ consecutive intervals $\left[0, z_{1}\right),\left[z_{1}, z_{2}\right), \ldots\left[z_{j}, z\right)$. We can view these intervals as urns, and if for example $X_{i}(\omega) \in\left[z_{l-1}, z_{l}\right)$, we will say that in this urn model the $j$-th point (or particle) has been placed in the $l$-th urn. It is well known that the probability to have $g_{0}$ particles in the first urn, $g_{1}$ particles in the second urn and so on, $g_{j-1}$ particles in the $j$-th and $g_{j}$ particles in the last $(j+1)$-th urn, is given by the multinomial formula

$$
\frac{j !}{g_{0} ! \ldots g_{j} !} p_{1}^{g_{0}} \ldots p_{j+1}^{g_{j}}
$$

where $0 \leq g_{i} \leq j, i=0, \ldots, j$ and $g_{0}+g_{1}+\cdots+g_{j}=j$.

It can directly be checked that,

$$
P\left(T_{1}>z_{1}, \ldots, T_{j}>z_{j}\right)=\sum_{\left(g_{0}, \ldots, g_{j}\right) \in E(0, j)} \frac{j !}{g_{0} ! \ldots g_{j} !} p_{1}^{g_{0}} \ldots p_{j+1}^{g_{j}} .
$$

This completes the proof of Lemmas 2.7.

The following lemma, which directly follows from Lemmas 2.6 and 2.7. gives a probabilistic interpretation of the Appell-Hessenberg function, $A_{j}\left(F_{z}(z) ; F_{z}\left(z_{1}-\right), \ldots\right.$, $\left.F_{z}\left(z_{j}-\right)\right)$.

Lemma 2.9 Let $0 \equiv z_{0}<z_{1}<z_{2}<\ldots<z_{j}<z$ be a sequence of positive real numbers and for a fixed $z$, let $0<T_{1} \leq T_{2} \leq \ldots \leq T_{j} \leq z$ be the consecutive points of an OS point process $\xi$, defined as in Definition 1.1. We have

$$
P\left(T_{1}>z_{1}, \ldots, T_{j}>z_{j}\right)=A_{j}\left(F_{z}(z) ; F_{z}\left(z_{1}-\right), \ldots, F_{z}\left(z_{j}-\right)\right),
$$

where $A_{j}\left(F_{z}(z) ; F_{z}\left(z_{1}-\right), \ldots, F_{z}\left(z_{j}-\right)\right)$ is defined as in Eq. 2.

We are now ready to prove Theorem 2.4. 
Proof of Theorem 2.4 Applying the formula of total probability, we have

$$
P(T \leq z, Y>y)=\sum_{j=1}^{\infty} P(\xi(0, z]=j) P(T \leq z, Y>y \mid \xi(0, z]=j)
$$

For the conditional probability on the right-hand side of Eq. 20, we have

$$
\begin{aligned}
& P(T \leq z, Y>y \mid \xi(0, z]=j) \\
& =P\left(\bigcup_{k=1}^{j}\left\{\left(\bigcap_{l=1}^{k-1}\left(h\left(T_{l}\right)>Y_{l}\right)\right) \cap\left(h\left(T_{k}\right)+y<Y_{k}\right)\right\}\right),
\end{aligned}
$$

where we assume that, for $k=1, \bigcap_{l=1}^{k-1}\left(h_{z}\left(T_{l}\right)>Y_{l}\right)$ is the sure event, i.e. $\bigcap_{l=1}^{0}\left(h\left(T_{l}\right)>Y_{l}\right)$ $\equiv \Omega$.

Continuing Eq. 21, we have

$$
P(T \leq z, Y>y \mid \xi(0, z]=j)=\sum_{k=1}^{j} P\left(\left(\bigcap_{l=1}^{k-1}\left(h\left(T_{l}\right)>Y_{l}\right)\right) \cap\left(h\left(T_{k}\right)+y<Y_{k}\right)\right)
$$

where we have used the fact that the events are disjoint. Indeed, if we take two events,

$$
\left(\bigcap_{l=1}^{k-1}\left(h\left(T_{l}\right)>Y_{l}\right)\right) \cap\left(h\left(T_{k}\right)+y<Y_{k}\right)
$$

and

$$
\left(\bigcap_{l=1}^{k+s-1}\left(h\left(T_{l}\right)>Y_{l}\right)\right) \cap\left(h\left(T_{k+s}\right)+y<Y_{k+s}\right)
$$

where $s \geq 1, k \geq 1$ and $s+k \leq j$, then it is easy to see that the events from Eqs. 23 and 24 are disjoint for $s \geq 1, k \geq 1$ and $s+k \leq j, y \geq 0$. In view of Eq. 22, we can equivalently rewrite equality (20) as

$$
P(T \leq z, Y>y)=P(\xi(0, z]=1) P\left(T_{1}<h_{z}^{-1}\left(Y_{1}-y\right)\right)+\sum_{j=2}^{\infty} P(\xi(0, z]=j) \sum_{k=1}^{j} P\left(G_{k}\right),
$$

where $\left(T_{1}<h_{z}^{-1}\left(Y_{1}-y\right)\right)$ is the event of ruin at the first claim with deficit at least $y$ and

$$
G_{k}=\left(\bigcap_{l=1}^{k-1}\left(T_{l}>h_{z}^{-1}\left(Y_{l}\right)\right)\right) \cap\left(T_{k}<h_{z}^{-1}\left(Y_{k}-y\right)\right), k=2,3, \ldots
$$


is the event of survival after the first $k-1$ claims have arrived and ruin at the $k$-th claim with deficit at least $y$. Let us now transform the probabilities in Eq. 25. By means of conditional probabilities, we have

$$
\begin{aligned}
& P\left(T_{1}<h_{z}^{-1}\left(Y_{1}-y\right)\right)=\int_{0}^{+\infty} P\left(T_{1}<h_{z}^{-1}\left(y_{1}-y\right)\right) f\left(y_{1}\right) d y_{1} \\
& =\int_{y}^{+\infty} P\left(T_{1}<h_{z}^{-1}\left(y_{1}-y\right)\right) f\left(y_{1}\right) d y_{1} \\
& =\int_{y}^{+\infty}\left(1-P\left(T_{1}>h_{z}^{-1}\left(y_{1}-y\right)\right)\right) f\left(y_{1}\right) d y_{1} \\
& \left.=\int_{y}^{+\infty}\left(1-P\left(\xi\left(0, h_{z}^{-1}\left(y_{1}-y\right)\right]=0\right)\right)\right) f\left(y_{1}\right) d y_{1}
\end{aligned}
$$

where the range of integration is $y_{1} \in[y,+\infty]$ since for $0<y_{1}<y, h_{z}^{-1}\left(y_{1}-y\right)=0$ and $\left(T_{1}<h_{z}^{-1}\left(y_{1}-y\right)\right) \equiv\left(T_{1}<0\right)$ becomes the impossible event $\emptyset$. Let us now simplify $P\left(G_{k}\right), k=2,3, \ldots$. We have

$$
\begin{aligned}
& P\left(G_{k}\right)=\int_{0 \leq y_{1} \leq \ldots \leq y_{k}} \ldots \int_{l=1} P\left(\left(\bigcap_{l=1}^{k-1}\left(T_{l}>h_{z}^{-1}\left(y_{l}\right)\right)\right) \cap\left(T_{k}<h_{z}^{-1}\left(y_{k}-y\right)\right)\right) f\left(y_{1}, \ldots, y_{k}\right) d y_{1} \ldots d y_{k} \\
& =\int_{\substack{0 \leq y_{1} \leq \ldots \leq y_{k-1} \leq y_{k}-y, y_{k-1} \leq h_{z}(z)}} P\left(\left(\bigcap_{l=1}^{k-1}\left(T_{l}>h_{z}^{-1}\left(y_{l}\right)\right)\right) \cap\left(T_{k}<h_{z}^{-1}\left(y_{k}-y\right)\right)\right) f\left(y_{1}, \ldots, y_{k}\right) d y_{1} \ldots d y_{k}
\end{aligned}
$$

where in the last equality in Eq. 27 we have cut off the domain of integration, $0 \leq y_{1} \leq \ldots \leq y_{k}$, where the conditional probability is 0 . Setting $C_{k}=$ $\left\{\left(y_{1}, \ldots, y_{k}\right): 0 \leq y_{1} \leq \ldots \leq y_{k-1} \leq y_{k}-y, y_{k-1} \leq h_{z}(z)\right\}$ and using the identity $P(A \cap$ $B)=P(A)-P(A \cap \overline{\bar{B}})$, from Eq. 27 we obtain

$$
\begin{aligned}
& P\left(G_{k}\right)=\int \ldots \int\left\{P\left[\bigcap_{C_{k}}^{k-1}\left(T_{l}>h_{z}^{-1}\left(y_{l}\right)\right)\right]\right. \\
& \left.-P\left[\left(\bigcap_{l=1}^{k-1}\left(T_{l}>h_{z}^{-1}\left(y_{l}\right)\right)\right) \cap\left(T_{k}>h_{z}^{-1}\left(y_{k}-y\right)\right)\right]\right\} f\left(y_{1}, \ldots, y_{k}\right) d y_{1} \ldots d y_{k}
\end{aligned}
$$

From Eqs. 25, 26 and 28, applying Lemma 2.9 to the probabilities on the right-hand side of Eq. 28 we obtain the asserted formula (6).

The following corollaries of Theorem 2.4 give explicitly formulas for the joint distribution of the ruin time and deficit and for the finite time probability of ruin, under an OS claim arrival process defined as in Definition 1.1. 
Corollary 2.10 In the case of discrete claim amounts $W_{1}, W_{2}, \ldots$ with joint probability mass function $P_{w_{1}, \ldots, w_{k}}=P\left(W_{1}=w_{1}, \ldots, W_{k}=w_{k}\right), k=1,2, \ldots$, we have

$$
\begin{aligned}
P(T \leq & , Y>y)=P(\xi(0, z]=1)\left(1-\sum_{w_{1}=1}^{m-1} P_{w_{1}}-\sum_{w_{1}=m}^{l} P_{w_{1}} \times P\left(\xi\left(0, h_{z}^{-1}\left(w_{1}-y\right)\right)=0\right)\right) \\
& +\sum_{j=2}^{l} P(\xi(0, z]=j) \sum_{k=1}^{j} \sum_{\left(w_{1}, \ldots, w_{k}\right) \in \tilde{C}_{k}} P_{w_{1}, \ldots, w_{k}} \\
& \times\left\{A_{k-1}\left(1 ; F_{z}\left(h_{z}^{-1}\left(w_{1}\right)-\right), \ldots, F_{z}\left(h_{z}^{-1}\left(w_{1}+\ldots+w_{k-1}\right)-\right)\right)\right. \\
& \left.-A_{k}\left(1 ; F_{z}\left(h_{z}^{-1}\left(w_{1}\right)-\right), \ldots, F_{z}\left(h_{z}^{-1}\left(w_{1}+\ldots+w_{k}-y\right)-\right)\right)\right\}
\end{aligned}
$$

where $m=[y]+1$,

$$
l=\left\{\begin{array}{l}
{\left[h_{z}(z)+y\right] \quad \text { if } h_{z}(z)+y \text { is not integer },} \\
h_{z}(z)+y-1 \quad \text { if } h_{z}(z)+y \text { is integer },
\end{array}\right.
$$

with $[\cdot]$ denoting the integer part, $\tilde{C}_{k}=\left\{\left(w_{1}, \ldots, w_{k}\right): 1 \leq w_{i}, i=1, \ldots, k, y<\right.$ $\left.w_{k}, w_{1}+\ldots+w_{k-1} \leq h_{z}(z)\right\}$, and $A_{k}\left(1 ; F_{z}\left(z_{1}-\right), \ldots, F_{z}\left(z_{k}-\right)\right)$ the classical Appell polynomials evaluated at $F_{z}(z)=1$ and defined as in Eq. 2 .

Let us note that Eq. 29 is an explicit and exact expression, involving only finite summations and is therefore appealing for numerical purposes. We now give some useful special cases of our main result.

Corollary 2.11 The following expression for the probability of ruin $P(T<z)$ follows directly from Theorem 2.4 .

$$
\begin{aligned}
P(T<z)= & P(T<z, Y>0) \\
= & P(\xi(0, z]=1) \int_{0}^{\infty}\left(1-A_{1}\left(1 ; h_{z}^{-1}\left(y_{1}\right)\right)\right) f\left(y_{1}\right) d y_{1} \\
& +\sum_{j=2}^{\infty} P(\xi(0, z]=j) \sum_{k=1}^{j} \int \ldots \int\left\{A_{D_{k}}\left(1 ; F_{z}\left(h_{z}^{-1}\left(y_{1}\right)-\right), \ldots, F_{z}\left(h_{z}^{-1}\left(y_{k-1}\right)-\right)\right)\right. \\
& \left.-A_{k}\left(1 ; F_{z}\left(h_{z}^{-1}\left(y_{1}\right)-\right), \ldots, F_{z}\left(h_{z}^{-1}\left(y_{k-1}\right)-\right), F_{z}\left(h_{z}^{-1}\left(y_{k}\right)-\right)\right)\right\} \\
& f\left(y_{1}, \ldots, y_{k}\right) d y_{k} \ldots d y_{1},
\end{aligned}
$$

where $D_{k}=\left\{\left(y_{1}, \ldots, y_{k}\right): 0<y_{1}<\ldots<y_{k-1} \leq y_{k}, y_{k-1} \leq h_{z}(z)\right\}$, and $A_{j}\left(1 ; F_{z}\left(z_{1}\right), \ldots, F_{z}\left(z_{j}\right)\right)$, are the classical Appell polynomials evaluated at $F_{z}(z)=1$ and defined as in Eq. 2 with $z_{1}=h_{z}^{-1}\left(y_{1}\right), \ldots, z_{j}=h_{z}^{-1}\left(y_{j}\right), j=0,1,2 \ldots$.

The following corollary of Theorem 2.4 provides a different and more general formula (in the sense of Definition 1.1) than the non-ruin formula (4.8) (preceded by (4.1) and (4.2)) obtained by Lefèvre and Picard (2011) for the particular case of an OS risk model with continuous $F_{z}(x)$. We note that formula (4.8) of Lefèvre and Picard (2011) is a sum of a product of two expectations, involving two indicator functions and a slightly different version of Appell polynomials, and therefore, is different in structure from the formula given 
next. In addition, allowing for discontinuous $F_{z}(x)$ (cf. Definition 1.1) affects the zeros of the Appell polynomials involved in formulas (6), (29), (30), (31) expressed through $F_{z}(.-)$. Recall that the latter are the left-hand side limits of $F_{z}($.$) at the points of discontinuities.$

Corollary 2.12 For the probability of non-ruin $P(T>z)$, assuming the OS claim arrival process from Definition 1.1, we have

$$
\begin{aligned}
P(T>z)= & P(\xi(0, z]=0) \\
+ & \sum_{j=1}^{\infty} P(\xi(0, z]=j)\left(1-\sum_{k=1}^{j} \int_{D_{k}} \ldots \int\left\{A _ { k - 1 } \left(1 ; F_{z}\left(h_{z}^{-1}\left(y_{1}\right)-\right),\right.\right.\right. \\
& \left.\ldots, F_{z}\left(h_{z}^{-1}\left(y_{k-1}\right)-\right)\right) \\
& \left.-A_{k}\left(1 ; F_{z}\left(h_{z}^{-1}\left(y_{1}\right)-\right), \ldots, F_{z}\left(h_{z}^{-1}\left(y_{k-1}\right)-\right), F_{z}\left(h_{z}^{-1}\left(y_{k}\right)-\right)\right)\right\} \\
& \left.f\left(y_{1}, \ldots, y_{k}\right) d y_{k} \ldots d y_{1}\right),
\end{aligned}
$$

Proof of Corollary 2.12 From Corollary 2.11, multiplying both sides of Eq. 30 by -1 and adding one on each side gives

$$
\begin{aligned}
& 1-P(T<z) \\
& =1-P(\xi(0, z]=1) \int_{0}^{\infty}\left(1-A_{1}\left(1 ; h_{z}^{-1}\left(y_{1}\right)\right)\right) f\left(y_{1}\right) d y_{1} \\
& -\sum_{j=2}^{\infty} P(\xi(0, z]=j) \sum_{k=1}^{j} \int \ldots \int\left\{A_{k-1}\left(1 ; F_{z}\left(h_{z}^{-1}\left(y_{1}\right)-\right), \ldots, F_{z}\left(h_{z}^{-1}\left(y_{k-1}\right)-\right)\right)\right. \\
& \left.-A_{k}\left(1 ; F_{z}\left(h_{z}^{-1}\left(y_{1}\right)-\right), \ldots, F_{z}\left(h_{z}^{-1}\left(y_{k-1}\right)-\right), F_{z}\left(h_{z}^{-1}\left(y_{k}\right)-\right)\right)\right\} f\left(y_{1}, \ldots, y_{k}\right) d y_{k} \ldots d y_{1},
\end{aligned}
$$

Applying the identity $1 \equiv \sum_{j=0}^{\infty} P(\xi(0, z]=j)$ to express the unity on the right-hand side of Eq. 32, after some elementary algebra of summing up the factors (one of which equal to 1) multiplying each of the terms $P(\xi(0, z]=j)$, we obtain

$$
\begin{aligned}
P(T>z)= & 1-P(T<z) \\
= & P(\xi(0, z]=0)+P(\xi(0, z]=1)\left(1-\int_{0}^{\infty}\left(1-A_{1}\left(1 ; h_{z}^{-1}\left(y_{1}\right)\right)\right) f\left(y_{1}\right) d y_{1}\right) \\
& +\sum_{j=2}^{\infty} P(\xi(0, z]=j)\left(1-\sum_{k=1}^{j} \int_{D_{k}} \ldots \int\left\{A _ { k - 1 } \left(1 ; F_{z}\left(h_{z}^{-1}\left(y_{1}\right)-\right), \ldots,\right.\right.\right. \\
& \left.F_{z}\left(h_{z}^{-1}\left(y_{k-1}\right)-\right)\right) \\
& \left.-A_{k}\left(1 ; F_{z}\left(h_{z}^{-1}\left(y_{1}\right)-\right), \ldots, F_{z}\left(h_{z}^{-1}\left(y_{k-1}\right)-\right), F_{z}\left(h_{z}^{-1}\left(y_{k}\right)-\right)\right)\right\} \\
& f\left(y_{1}, \ldots, y_{k}\right) d y_{k} \ldots d y_{1},
\end{aligned}
$$


The asserted Eq. 31 now follows noting that the second term on the right-hand side of Eq. 33 can be added to the sum, for $j=1$.

Let us note that Corollaries 2.11 and 2.12 generalize previous ruin probability formulas obtained for the Poisson case, e.g., formula (34) of Ignatov and Kaishev (2000), formula (7) of Ignatov et al. (2001), and formula (1) of Ignatov and Kaishev (2004). Numerical properties and relations between the latter formulas are considered in Dimitrova et al. (2016). It is worth also noting that since the Poisson process is at the same time an OS process and a process with independent increments, both formula (6) (cf Theorem 2.4) and formula (28) of Ignatov and Kaishev (2016), (cf Corollary 3.7 therein) cover this special case but formula (6) is simpler in structure compared to formula (28) of Ignatov and Kaishev (2016).

\section{$3 P(T \leq z, Y>y)$ for Some Special Cases of the OS Claim Arrival Process $\xi$}

In this section we consider applications of our main result given by Theorem 2.4, which cover the two important special cases of OS claim arrival processes, i.e., when $F_{z}(x)$ is assumed continuous, or discontinuous. In the first case there are three models considered previously in the literature, which arise under the assumption of stationary transition probabilities of the OS process, namely, the (mixed) Poisson, negative binomial and binomial models (see e.g. Crump (1975) and Lefèvre and Picard (2011, 2014). In the second case of $F_{z}(x)$ with possible jump discontinuities, we consider purely discrete time OS point processes with claims arriving at some fixed instants, forming a sequence of positive real numbers, and general OS point processes with arrivals both at fixed and random instants. To the best of our knowledge such OS claim arrival models have not been considered in the risk and ruin literature.

\section{1 $P(T \leq z, Y>y)$ for OS Processes Generated by a Continuous cdf $F_{z}(x)$}

When $\xi$ has stationary transition probabilities, i.e., when $P(\xi(0, s]=j \mid \xi(0, t]=i)$, with $t<s$ and $i \leq j$, only depends on $s-t$, it has been established by Crump (1975) that the OS process $\xi$ must be one of the three processes: either a homogeneous Poisson process or a linear birth process with immigration, or a linear death process. We will revisit these three special cases of OS claim arrivals which have also been considered by Lefèvre and Picard (2011, 2014), who give particular expressions for the non-ruin probability. The latter expressions are directly covered and generalized, applying Corollary 2.12 and Theorem 2.4, i.e., evaluating $P(T>z)$ and $P(T \leq z, Y>y)$, substituting in Eqs. 6 and 31 the specific expressions for $P(\xi(0, s]=j)$ and $F_{z}(z)$, for each of the three cases, as follows.

(a) If $\xi$ is a Poisson process with rate $\lambda$, then $F_{z}(x)=x / z, 0 \leq x \leq z$, which corresponds to $X \equiv 1$ and $\Lambda(z) \equiv \lambda z$ in representation (1). The latter holds also in the mixed Poisson case, randomizing $\lambda$;

(b) if $\xi$ is a linear birth process with immigration, with birth rate $b>0$ and immigration rate $a \geq 0$, then $\xi(0, z]$ has a negative binomial distribution, with parameters, $a / b$ and $1-e^{-\bar{b} z}$ and $F_{z}(x)=\left(e^{b x}-1\right) /\left(e^{b z}-1\right)$;

(c) if $\xi$ is the number of deaths in a linear death process with initial population size, $\rho$ (positive integer), and death rate $d>0$, then $\xi(0, z]$ has a binomial distribution with parameters, $\rho$ and $1-e^{-d z}$ and $F_{z}(x)=\left(1-e^{-b x}\right) /\left(1-e^{-b z}\right)$. 


\section{2 $P(T \leq z, Y>y)$ for OS Processes Generated by a cdf $F_{z}(x)$ with Possible Jump Discontinuities}

Let us note that OS processes defined as in Definition 1.1 form a new interesting class, whose characterization is outside the scope of this paper and will be considered separately. Here, our purpose will be to give examples of such OS processes of relevance to modeling insurance claim arrivals in the context of ruin.

First we consider the case when there are claim counts, $\xi_{1}, \xi_{2}, \ldots$ at some fixed instants $0<t_{1}<t_{2}<\ldots$ which could be observed regularly e.g. monthly or annually, for say, $j$ periods of time. Such data, called longitudinal (or panel) data is typically collected by insurance companies at individual and portfolio levels. In such cases it is common to seek for appropriate discrete distributions in order to model the marginal and the joint distribution of the r.v.s $\xi_{1}, \ldots, \xi_{j}$. Different count data models have been proposed, based on integervalued (count) time series and random effect Poisson or negative binomial distributions (see e.g., Boucher et al. 2007, Denuit et al. 2007 and Shi and Valdez 2014). Our purpose here will be to demonstrate, based on a simple example, that the general OS processes introduced by Definition 1.1 can also be used to model claim counts data in the context of ruin probability.

Define the sequence of independent Poisson distributed random variables, $\xi_{i} \in \mathcal{P}\left(\mu_{i}\right)$, $i=1,2, \ldots$ where $\xi_{1}$ is attached to $t_{1}, \xi_{2}$ to $t_{2}$ and so on, and the point (claim count) process $\xi(0, x]=\xi_{1} \mathbb{1}_{(0, x]}\left(t_{1}\right)+\ldots+\xi_{j} \mathbb{1}_{(0, x]}\left(t_{j}\right)$, where

$$
\mathbb{1}_{(0, x]}(u)=\left\{\begin{array}{l}
0 \quad \text { if } u>x, \\
1 \text { if } 0<u \leq x .
\end{array}\right.
$$

We will define its underlying cdf $F_{z}(x)$, for $z \geq t_{1}$ since for $0<z<t_{1}$, with probability one there will be no claims in the interval $(0, z]$ and it does not make sense to define $F_{z}(x)$, also because it does not appear in formula (6) of Theorem 2.4 and its corollaries. Assume that $t_{j} \leq z<t_{j+1}$. Then

$$
F_{z}(x)=\left\{\begin{array}{cc}
0 & \text { for } x<t_{1}, \\
\frac{\mu_{1}}{\mu_{1}+\ldots+\mu_{j}} & \text { for } t_{1} \leq x<t_{2}, \\
\ldots & \\
\frac{\mu_{1}+\ldots+\mu_{j-1}}{\mu_{1}+\ldots+\mu_{j}} & \text { for } t_{j-1} \leq x<t_{j}, \\
1 & \text { for } \min \left(z, t_{j}\right) \leq x
\end{array}\right.
$$

and it can be seen that $\xi$ is an OS process, where the claim clusters, $\xi_{1}, \ldots, \xi_{j}$ are concentrated at the points $0<t_{1}<\ldots<t_{j}$, respectively. Therefore, one can directly apply Theorem 2.4 and its corollaries to obtain various ruin related quantities. We will demonstrate how the non-ruin probability can be computed using Corollary 2.12 , based on the following simple example.

Example 3.1 Let $0<t_{1}<t_{2} \leq z$. Then from Eq. 34 for $F_{z}(x)$, we have

$$
F_{z}(x)=\left\{\begin{array}{cc}
0 & \text { for }-\infty<x<t_{1}, \\
\frac{\mu_{1}}{\mu_{1}+\mu_{2}} & \text { for } t_{1} \leq x<t_{2} \\
1 & \text { for } t_{2} \leq x<+\infty
\end{array}\right.
$$

Take the premium income function to be,

$$
h_{z}(x)=\left\{\begin{array}{l}
0.5 \quad \text { for } x<t_{1}, \\
1.5 \text { for } t_{1} \leq x<t_{2}, \\
2.5 \quad \text { for } t_{2} \leq x,
\end{array}\right.
$$


and the partial claim sums, $Y_{1}, Y_{2}, \ldots$ to be the integers $1,2, \ldots$, respectively. Applying Eq. 31 of Corollary 2.12, for the non-ruin probability, $P(T>z)$, we have

$$
\begin{aligned}
P(T>z)= & P(\xi(0, z]=0)+P(\xi(0, z]=1)\left(1-\left[A_{0}(1)-A_{1}\left(1 ; F_{z}\left(h_{z}^{-1}(1)-\right)\right)\right]\right) \\
& +P(\xi(0, z]=2)\left(1-\left[A_{0}(1)-A_{1}\left(1 ; F_{z}\left(h_{z}^{-1}(1)-\right)\right)+A_{1}\left(1 ; F_{z}\left(h_{z}^{-1}(1)-\right)\right)\right.\right. \\
& \left.\left.-A_{2}\left(1 ; F_{z}\left(h_{z}^{-1}(1)-\right), F_{z}\left(h_{z}^{-1}(2)-\right)\right)\right]\right) \\
= & P(\xi(0, z]=0)+P(\xi(0, z]=1)\left(1-\left[1-A_{1}\left(1 ; F_{z}\left(t_{1}-\right)\right)\right]\right) \\
& +P(\xi(0, z]=2)\left(1-\left[1-A_{2}\left(1 ; F_{z}\left(t_{1}-\right), F_{z}\left(t_{2}-\right)\right)\right]\right) \\
= & \exp \left(-\left(\mu_{1}+\mu_{2}\right)\right)+\left(\mu_{1}+\mu_{2}\right) \exp \left(-\left(\mu_{1}+\mu_{2}\right)\right)\left(1-\left[1-A_{1}(1 ; 0)\right]\right) \\
& +\frac{\left(\mu_{1}+\mu_{2}\right)^{2}}{2 !} \exp \left(-\left(\mu_{1}+\mu_{2}\right)\right)\left(1-\left[1-A_{2}\left(1 ; 0, \frac{\mu_{1}}{\mu_{1}+\mu_{2}}\right)\right]\right) \\
= & \exp \left(-\left(\mu_{1}+\mu_{2}\right)\right)\left(1+\mu_{1}+\mu_{2}+\frac{\mu^{2}}{2}+\mu_{1} \mu_{2}\right)
\end{aligned}
$$

where in the last equality we have used the fact that, $A_{1}(1 ; 0)=1$ and that $A_{2}\left(1 ; 0, \frac{\mu_{1}}{\mu_{1}+\mu_{2}}\right)=\left(1-\frac{\mu_{1}^{2}}{\left(\mu_{1}+\mu_{2}\right)^{2}}\right)$, (c.f. Eq. 2).

Remark 3.2 For simplicity and practical relevance, we have assumed in Eq. 35 that premiums are collected at the times $0, t_{1}, t_{2}$, but since $t_{1}, t_{2}$ are also the instants of the claim arrivals $\xi_{1}$ and $\xi_{2}$, the last expression in Eq. 36 does not depend on $t_{1}, t_{2}$ and $z$. Let us note that, in general, $P(T>z)$ will depend on the instants $t_{1}<t_{2}<\ldots<t_{j}<z$. To simplify the calculations, we have also assumed unit claim amounts, but in general, the expression for $P(T>z)$ will be more complex, involving the multiple integration with respect to the joint density $f\left(y_{1}, \ldots, y_{k}\right)$, following Eq. 31 .

Second, consider an OS point process, $\xi$ with a continuous component and a pure jump component in the underlying cdf, $F_{z}(x)$, i.e., $\xi(0, x]=\eta(0, x]+\xi_{1} \mathbb{1}_{(0, x]}\left(t_{1}\right)+\ldots+$ $\xi_{j} \mathbb{1}_{(0, x]}\left(t_{j}\right)$, where $\eta(0, x]$ is a Poisson process with unit rate, defined on $(0, \infty]$ and independent of the Poisson random variables, $\xi_{1}, \ldots, \xi_{j}, \xi_{i} \in \mathcal{P}\left(\mu_{i}\right), i=1, \ldots j$, assumed also mutually independent. By construction, $\xi$ is an OS process with independent increments. It could be particularly suitable for applications, especially when data comes from two (or more) independent insurance portfolios (lines of business), among which one with claim frequency data at fixed instants $0<t_{1}<t_{2}<\ldots<t_{j}$ (e.g. annual observations) and a second one with data at policy level of the instants of claiming. In view of the Solvency II requirements, it would be instructive to be able to evaluate the probability of non-ruin in a finite time interval, $(0, z]$, due to claims coming from all lines of business. Without loss of generality, we will illustrate this, based on the following example.

Example 3.3 Let $0<t_{1}<t_{2} \leq z$. Then, the cumulative intensity function, $\Lambda(x)$ is

$$
\Lambda(x)=\left\{\begin{array}{cc}
x & \text { for } 0<x<t_{1}, \\
x+\mu_{1} & \text { for } t_{1} \leq x<t_{2} \\
x+\mu_{1}+\mu_{2} & \text { for } t_{2} \leq x \leq z
\end{array}\right.
$$


and the related cdf

$$
F_{z}(x)=\left\{\begin{array}{cc}
0 & \text { for }-\infty<x<0, \\
x /\left(z+\mu_{1}+\mu_{2}\right) & \text { for } 0 \leq x<t_{1}, \\
\left(x+\mu_{1}\right) /\left(z+\mu_{1}+\mu_{2}\right) & \text { for } t_{1} \leq x<t_{2}, \\
\left(x+\mu_{1}+\mu_{2}\right) /\left(z+\mu_{1}+\mu_{2}\right) & \text { for } t_{2} \leq x \leq z, \\
1 & \text { for } z<x<+\infty
\end{array}\right.
$$

As in Example 3.1, take the premium income function to be, given by Eq. 35 and the partial claim sums, $Y_{1}, Y_{2}, \ldots$ to be the integers 1,2, ., respectively. Applying Eq. 31 of Corollary 2.12, similarly as in Eq. 36, we have

$$
\begin{aligned}
P(T>z)= & P(\xi(0, z]=0)+P(\xi(0, z]=1) A_{1}\left(1 ; F_{z}\left(h_{z}^{-1}(1)-\right)\right) \\
& +P(\xi(0, z]=2) A_{2}\left(1 ; F_{z}\left(h_{z}^{-1}(1)-\right), F_{z}\left(h_{z}^{-1}(2)-\right)\right) \\
= & \exp \left(-\left(z+\mu_{1}+\mu_{2}\right)\right)+\left(z+\mu_{1}+\mu_{2}\right) \exp \left(-\left(z+\mu_{1}+\mu_{2}\right)\right) A_{1}\left(1 ; \frac{t_{1}}{z+\mu_{1}+\mu_{2}}\right) \\
& +\frac{\left(z+\mu_{1}+\mu_{2}\right)^{2}}{2 !} \exp \left(-\left(z+\mu_{1}+\mu_{2}\right)\right) A_{2}\left(1 ; \frac{t_{1}}{z+\mu_{1}+\mu_{2}}, \frac{t_{2}+\mu_{1}}{z+\mu_{1}+\mu_{2}}\right) \\
= & \exp \left(-\left(z+\mu_{1}+\mu_{2}\right)\right)\left(1+z\left(1+\mu_{1}+\mu_{2}+\frac{z}{2}-t_{1}\right)+t_{1} t_{2}-\frac{t_{2}^{2}}{2}-\mu_{1} t_{2}\right. \\
& \left.+\mu_{1} \mu_{2}+\frac{\mu_{2}^{2}}{2}-\mu_{2} t_{1}+\mu_{1}+\mu_{2}-t_{1}\right),
\end{aligned}
$$

where in the last equality we have used Eq. 2 to evaluate $A_{1}\left(1 ; \frac{t_{1}}{z+\mu_{1}+\mu_{2}}\right)$ and $A_{2}\left(1 ; \frac{t_{1}}{z+\mu_{1}+\mu_{2}}, \frac{t_{2}+\mu_{1}}{z+\mu_{1}+\mu_{2}}\right)$.

Remark 3.4 Note that in contrast to Eq. 36 from Example 3.1, expression (37), depends on the instants $t_{1}, t_{2}, z$, which is due to the continuous time component, $\eta$, in $\xi$.

The last expression in Eq. 37, can be verified through direct but tedious calculations, see Dimitrova et al. (2014).

Open Access This article is distributed under the terms of the Creative Commons Attribution 4.0 International License (http://creativecommons.org/licenses/by/4.0/), which permits unrestricted use, distribution, and reproduction in any medium, provided you give appropriate credit to the original author(s) and the source, provide a link to the Creative Commons license, and indicate if changes were made.

\section{References}

Aurzada F, Doering L, Savov M (2013) Small time Chung-type LIL for Lévy processes. Bernoulli 19(1):115136

Berg M, Spizzichino F (2000) Time-lagged point processes with the order-statistics property. Math Meth Oper Res 2000(51):301-314

Bernyk V, Dalang RC, Peskir G (2008) The law of the supremum of stable Lévy processes with no negative jumps. Ann Probab 36:1777-1789

Bertoin J, Doney RA, Maller RA (2008) Passage of Lévy processes across power law boundaries at small times. Ann Probab 36(1):160-197 
Borovkov AA (1964) On the first passage time for one class of processes with independent increments. Theor Probab Appl 10:331-334

Boucher JP, Denuit M, Guillén M (2007) Risk classification for claim counts: A comparative analysis of various zero inflated mixed Poisson and hurdle models. North American Actuarial Journal 11(4):110131

Bühlmann H (1970) Mathematical Methods in Risk theory. Springer, Heidelberg

Crump KS (1975) On point processes having an order statistics structure. Sankhyā:The Indian Journal of Statistics Series A 37(3):396-404

Denuit M, Maréchal X, Pitrebois S, Walhin J-F (2007) Actuarial modelling of claim counts: Risk classification, credibility and bonus-malus systems. Wiley, West Sussex

De Vylder FF, Goovaerts MJ (1999a) Inequality extensions of Prabhu's formula in ruin theory. Insurance Math Econom 24:249-271

De Vylder FF, Goovaerts MJ (1999b) Homogeneous risk models with equalized claim amounts. Insurance Math Econom 26:223-238

Dimitrova DS, Ignatov ZG, Kaishev VK (2014) Ruin and deficit under claim arrivals with the order statistics property. preprint. Available at:. https://www.researchgate.net/publication/306364689_Ruin_and_deficit_ under_claim_arrivals_with_the_order_statistics_property

Dimitrova DS, Kaishev VK, Zhao S (2016) On the evaluation of finite-time ruin probabilities in a dependent risk model. Appl Math Comput 275:268-286

Doney RA (1991) Hitting probabilities for spectrally positive Lévy processes. J London Math Soc 44(3):556576

Doney RA, Kyprianou AE (2006) Overshoots and undershoots of Lévy process. Ann Appl Probab 16:91-106

Eder I, Klüppelberg C (2009) The first passage event for sums of dependent Lévy processes with applications to insurance risk. Ann Appl Probab 19(6):2047-2079

Feigin PD (1979) On the characterization of point processes with the order statistic property. J Appl Probab 16:297-304

Garrido J, Morales M (2006) On The Expected Discounted Penalty function for Lévy Risk Processes. North American Actuarial Journal 10(4):196-216

Gerber HU, Shiu ESW (1997) The joint distribution of the time of ruin, the surplus immediately before ruin, and the deficit at ruin. Insurance Math Econom 21:129-137

Gerber HU, Shiu ESW (1998) On the time value of ruin. North American Actuarial Journal 2:48-78

Haccou P, Jagers P, Vatutin V.A (2005) Branching processes: Variation, growth, and extinction of populations. Cambridge University Press, Cambridge

Holmes PT (1971) On a property of Poisson process. Sankhyā:The Indian Journal of Statistics Series A 33(1):93-98

Huang WJ, Shoung JM (1994) On a study of some properties of point processes. Sankhyā:The Indian Journal of Statistics Series A 56(1):67-76

Huzak M, Perman M, Sikic H, Vondracek Z (2004) Ruin probabilities and decompositions for general perturbed risk processes. Ann Appl Probab 14(3):1378-1397

Ignatov ZG, Kaishev VK (2000) Two-sided Bounds for the Finite-time Probability of Ruin. Scand Actuar J 2000(1):46-62

Ignatov ZG, Kaishev VK (2004) A finite-time ruin probability formula for continuous claim severites. J Appl Probab 41(2):570-578

Ignatov ZG, Kaishev VK (2006) On the infinite-horizon probability of (non)ruin for integer-valued claims. J Appl Probab 43:535-551

Ignatov ZG, Kaishev VK (2016) First crossing time, overshoot and Appell-Hessenberg type functions. Stochastics 88(8):1240-1260

Ignatov ZG, Kaishev VK, Krachunov RS (2001) An Improved Finite-time Ruin Probability Formula and its "Mathematica" Implementation. Insurance Math Econom 29(3):375-386

Kallenberg O (1976) Random measures. Akademie-Verlag, Berlin

Kendall D (1949) Stochastic Processes and Population Growth. J R Stat Soc Ser B 11(2):230-282

Kou SG, Wang H (2003) First passage times of a jump diffusion process. Adv Appl Probab 35:504-531

Klüppelberg C, Kyprianou AE, Maller RA (2004) Ruin probabilities and overshoots for general Lévy insurance risk processes. Ann Appl Probab 14(4):1766-1801

Landriault D, Willmot GE (2009) On the joint distributions of the time to ruin, the surplus prior to ruin, and the deficit at ruin in the classical risk model. North American Actuarial Journal 13:252-270

Lefèvre C., Loisel S (2009) Finite-time ruin probabilities for discrete, possibly dependent, claim severities. Methodol Comput Appl Probab 11(3):425-441

Lefèvre C, Picard P (2011) A new look at the homogeneous risk model. Insurance Math Econom 49:512-519 
Lefèvre C, Picard P (2014) Ruin Probabilities for Risk Models with Ordered Claim Arrivals. Methodol Comput Appl Probab 16:885-905

Liberman U (1985) An order statistic characterization of the Poisson renewal process. J Appl Probab 22:717722

Nawrotzki K (1962) Ein Grenzwertsatz fr homogene zufllige Punktfolgen (Verallgemeinerung eines Satzes von A. Rényi). Math Nachricht 24:201-217

Peskir G (2007) The law of the passage times to points by a stable Lévy process with no-negative jumps. Research Report No 15, Probability and Statistics Group School of Mathematics, The Univ. Manchester

Picard P, Lefèvre C (1997) The probability of ruin in finite time with discrete claim size distribution. Insurance Math Econom 20(3):260-261

Puri PS (1982) On the characterization of point processes with the order statistic property without the moment condition. J Appl Probab 19:39-51

Savov M (2009) Small time two-sided LIL behavior for Lévy processes at zero. Probab Theory and Related Fields 144(1-2):79-98

Sendova KP, Zitikis R (2012) The order statistic claim process with dependent claim frequencies and severities. J Stat Theory Practice 6(4):597-620

Shi P, Valdez E (2014) Longitudinal modeling of insurance claim counts using jitters. Scandinavian Actuarial Journal 2014(2):159-179

Vein R, Dale P (1999) Determinants and their applications in mathematical physics. Springer-Verlag, New York

Westcott M (1973) Some remarks on a property of the Poisson process. Sankhyā:The Indian Journal of Statistics Series A 35:29-34

Willmot GE (1989) The total claims distribution under inflationary conditions. Scand Actuar J 1:1-12

Yang H, Zhang L (2001) Spectrally negative Levy processes with applications in risk theory. Adv Appl Probab 33(1):28191

Zolotarev VM (1964) The first passage time of a level and the behavior at infinity for a class of processes with independent increments. Theor Probab Appl 9:653-664 